\title{
Brief Training to Modify the Breadth of Attention Influences the Generalisation of Fear
}

\author{
Mohith M. Varma ${ }^{1} \cdot$ Riddhi J. Pitliya ${ }^{1} \cdot$ Tomislav D. Zbozinek $^{2} \cdot$ Tomer Shechner $^{3} \cdot$ Tom J. Barry $^{1,4}$ (I)
}

Accepted: 16 September 2020 / Published online: 1 October 2020

(c) The Author(s) 2020

\begin{abstract}
Background Generalisation of fear from dangerous to safe stimuli is an important process associated with anxiety disorders. However, factors that contribute towards fear (over)-generalisation remain poorly understood. The present investigation explored how attentional breadth (global/holistic and local/analytic) influences fear generalisation and, whether people trained to attend in a global vs. local manner show more or less generalisation.

Methods Participants $(N=39)$ were shown stimuli which comprised of large 'global' letters and smaller 'local' letters (e.g. an $F$ comprised of $A$ s) and they either had to identify the global or local letter. Participants were then conditioned to fear a face by pairing it with an aversive scream (75\% reinforcement schedule). Perceptually similar, but safe, faces, were then shown. Self-reported fear levels and skin conductance responses were measured.

Results Compared to participants in Global group, participants in Local group demonstrated greater fear for dangerous stimulus (CS +) as well as perceptually similar safe stimuli.

Conclusions Participants trained to attend to stimuli in a local/analytical manner showed higher magnitude of fear acquisition and generalisation than participants trained to attend in a global/holistic way. Breadth of attentional focus can influence overall fear levels and fear generalisation and this can be manipulated via attentional training.
\end{abstract}

\section{Introduction}

Fear is adaptive as it enables us to anticipate and react to potentially dangerous stimuli in our environment based on our previous experiences with dangerous stimuli. However, fear can be maladaptive if it is expressed in the presence of safe stimuli that only merely resemble a previously fear-evoking stimulus. This process of enhanced fear

Mohith M. Varma, and Riddhi J. Pitliya are co-first authors and contributed equally to this work

Tom J. Barry

tom.barry@kcl.ac.uk; tjbarry@hku.hk

1 Department of Psychology, The University of Hong Kong, 6.58 Jockey Club Tower, Pokfulam Road, Hong Kong, People's Republic of China

2 Division of Humanities and Social Sciences, California Institute of Technology, Pasadena, CA, USA

3 School of Psychological Sciences and the Integrated Brain and Behavior Research Center, University of Haifa, Haifa, Israel

4 Institute of Psychiatry, Psychology and Neuroscience, King's College London, London, UK generalisation is central to the aetiology and maintenance of anxiety and traumatic disorders (Dunsmoor \& Paz 2015; Dymond et al. 2015; Struyf et al. 2015). However, the process by which fear generalisation occurs and the factors that contribute towards individual differences in generalisation remain poorly understood.

Early (Hovland 1937) and contemporary fear generalisation investigations (e.g., Dunsmoor et al. 2009; Lissek et al. 2008; Struyf et al. 2017; Vervliet et al. 2010) have consistently shown that the extent to which fear generalises from initial conditioning experiences is influenced by a person's ability to perceptually discriminate between similar-looking stimuli (Ginat-Frolich et al. 2019,2017; Struyf et al. 2017; Zaman et al. 2019). For example, if a person were to meet a new, blonde-haired person conditional stimulus; (CS) who embarrassed them unconditional stimulus; (US), it may be adaptive to fear and avoid this person in future; however, if one began to fear all blonde people, or indeed all other people (generalisation stimuli; GSs), then this may become impairing. Heightened generalisation of fear has therefore been associated with the presence of elevated anxious symptoms and anxiety disorder diagnoses (Ahrens et al. 2015, 2016; Kaczkurkin et al. 2017; Laufer et al. 2016; Lissek 
et al. 2014) and has been found amongst people at risk of anxiety disorders, such as adolescents (Schiele et al. 2016). Individual differences in fear generalization are also thought to be driven by the extent to which a person can discriminate between a CS and its associated GSs (Holt et al. 2014; Laufer et al. 2016; Struyf et al. 2017; Zaman et al. 2019).

Successful discrimination between two similar stimuli requires a person to focus their attention on the distinctive features of a given stimulus whilst ignoring other, common features (Barry et al. 2016a, b, 2017). Individuals with impaired perceptual discrimination ability experience elevated anxiety levels and increased fear to both aversive and safe stimuli (Lissek et al. 2014; Staples-Bradley et al. 2018; Struyf et al. 2017). Researchers have also found that instructing people to direct their attentional focus (via verbal instruction) to similar features between a fear-evoking and a neutral stimulus increases fear generalisation between these stimuli (Vervliet et al. 2010). Also, individual differences in the extent to which people focus their attention on specific stimuli (e.g., threats vs. neutral stimuli) has been associated with differences in fear generalisation (Baker et al. 2019). Attention is thought to differ between individuals in terms of both the location of its focus (where one attends to) and its breadth (whether it is broad/global or narrow/local; Yoon et al. 2015). The present investigation provides the first examination of attentional breadth to fear generalisation.

Attention breadth is commonly described as global (holistic) and local (detail-oriented), which respectively refer to attention to the whole or parts of a composite stimulus (Navon 1977; Pomerantz 1983). These tendencies are thought to differ between people such that some people tend to exhibit a global preference, whereas others tend to focus their attention in a more local style (Navon 1977). People with a holistic/global attentional style may have a weakened ability to focus on the specific local features that distinguish similar stimuli from one another. Indeed, in the processing of facial stimuli, eye-tracking studies have demonstrated that global attention is associated with a focus of attention on the centre of faces, resulting in reduced ability to discriminate between facial stimuli, compared to a local attentional style where the features of the faces are scanned and processed (Chen et al. 2002; T. Chuk et al. 2014). It may be that people with a global attentional style therefore show enhanced generalisation of fear because of their failure to discriminate between dangerous stimuli and non-dangerous stimuli that are perceptually similar. However, it is also of note that there is an association between anxious states and focused attention to local perceptual features (Derryberry \& Reed 1998, 2002) and so it might also be possible that people with a local or detail-oriented attentional style experience greater anxiety than people with a more global attentional style, and so may show a broad fear for a range of stimuli.

The current study examined the relations between attentional breadth and fear generalisation by experimentally manipulating the breadth of participants' attention prior to fear conditioning in order to examine its effects on fear generalisation. Participants were trained to attend to stimuli in a global or local manner in a modified version of the classic Navon task (Navon 1977). This task is comprised of presentations of large 'global' letters made up of smaller 'local' letters (e.g. an $F$ composed of $A$ s; see Fig. 1). Participants in the Global attention training group were asked to identify
Fig. 1 Example stimuli from the attention training protocol (top; Global letter $=F$, Local letter $=\mathrm{A}$ ) and generalisation protocol (bottom). Conditional stimuli (CS) are shown along with perceptual morphs of these stimuli: GS1, 80\% CS +/20\% CS-; GS2, 60\%/40\%; GS3, $40 \% / 60 \%$; GS4, $20 \% / 80 \%$. The CS that was paired with the unconditional stimulus ( $\mathrm{CS}+$ ) and the CS that was not paired with the unconditional stimulus (CS-) were counterbalanced between participants. When the unconditional stimulus (scream) was presented, the CS was shown with a surprised expression
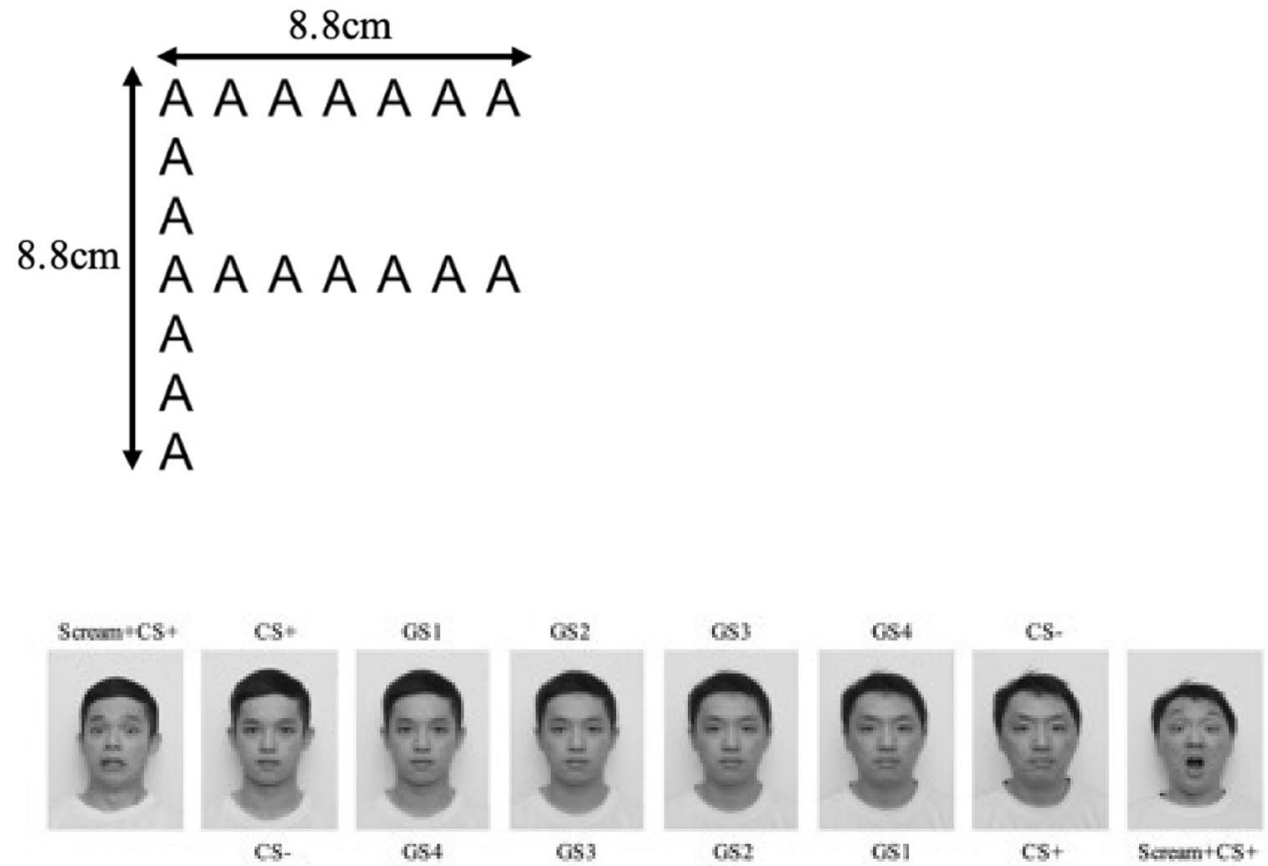

C\&!
$\mathrm{Cs}$
Scram+CS+ 
and press a keyboard button corresponding with the global letter while those in the Local training group were asked to identify the local letter. Following attention training, participants underwent a fear-conditioning and generalisation paradigm whilst the extent to which they perspired (skin conductance response) and reported fear in anticipation of the US was quantified. That the modification of attention occurred prior to fear conditioning is reminiscent of the kinds of differences in attentional breadth that are associated with negative moods and trait anxiety, which are thought to precede the emergence of anxiety disorders (Derryberry \& Reed 1998, 2002; Finucane \& Power 2010; Gasper \& Clore 2002). As such, this procedure was designed to model the process by which individual differences in attentional scope could influence the way that people respond to aversive events that happen in their life and the extent to which people subsequently generalize their fear from these events to other perceptually related events. Data and analysis scripts of the present study are available at: https://osf.io/ns5qt /?view_only=b08f1c31caa94a23b14766cdc9295d0f.

\section{Methods}

\section{Participants}

Thirty-nine students (14 males, 25 females; age range $=18-26$ years) from the first authors' institution were recruited through advertisements placed on bulletin boards across campus and through the mass email service to students. Estimating a sample of this size was based on earlier studies in this area that have employed expectancy rating and skin conductance responses as dependent variables $(N=32$; Vervliet et al. 2010) and such sample size is well powered (95\%) to detect effects in the small-to-moderate range $\left(\eta_{\mathrm{G}}{ }^{2}=0.10-0.20\right)$ in a repeated measures ANOVA with two groups. Participants were selected if they could read Traditional Chinese characters-for the purposes of reading the questionnaire measures-and, given the nature of the aversive stimulus used in the experiment, if they were not pregnant and had no ongoing heart problems or hearing impairments. All participants were students in an Englishlanguage degree programme and so were presumed to be familiar reading English characters. Participants could correct their vision with eyeglasses or contact lenses if necessary. The procedure received ethical approval from the first author's institution (EA1703042). All participants provided informed consent prior to participation and were debriefed after the experiment. Participants entered a cash-prize luckydraw or received course credit for their participation. Participants were randomly assigned to either the Global $(n=19)$ or Local $(n=20)$ training groups.

\section{Apparatus}

\section{Self-Report Measures}

Depression, anxiety and stress severity were measured using the 42-item, self-report Depression and Anxiety Stress Scale (DASS; Lovibond \& Lovibond 1995). Participants rated, on $0-3$ scales $(0=$ Does not apply to me at all; $3=$ Applies to me very much, or most of the time), to what extent each of the items was true of them in the past week. The total score and scores for all subscales were measured. Internal consistency of the DASS total score and sub-scales was good ( $\alpha=0.96$; depression, $\alpha=0.94$; anxiety, $\alpha=0.89$; stress, $\alpha=0.91)$.

\section{Attention Training Protocol}

The task was programmed and presented in Inquisit five (2016). Adapted from the Navon Task (1977), the stimuli comprised of a global letter made up of smaller local letters (see Fig. 1). The global and local letters used were A, F, H and K. A total of 12 stimuli were used such that every combination of global and local was created (e.g., an $A$ made of $F \mathrm{~s}$, an $F$ made of As, a $H$ made of $A$ s etc.). In each trial, participants were shown a fixation cross (' + ') at the center of the screen for $500 \mathrm{~ms}$, followed by a single stimulus which was presented until a response was made, marking the end of a trial. The inter-trial interval was fixed at $100 \mathrm{~ms}$. Depending on their group designation, prior to each block of trials participants were told whether they were to identify the global or local letter in the trials within that block. In global blocks, participants were asked to identify the global letter, and in local blocks participants were asked to identify the local letter. Each block comprised of 48 trials of two stimuli with opposing global and local letters (e.g., an $F$ made of $A$ s and an $A$ made of $F$ s) presented 24 times each in random order. During each stimulus presentation, participants made a choice between two responses: either the global or local letters. For example, in a global block, if an $F$ made of $A$ s was shown, participants could press $F$ or $A$ but a correct response would be to press $F$. If the same stimulus was shown in a local block, participants should press $A$. Two practice blocks (with global/local combinations of $P$ and $\mathrm{Y})$ and six test blocks were presented to each participant.

The Global group was only presented with global blocks, whereas, the Local group was only presented with local blocks. If participants in the training groups made an incorrect response, an error message with a cross ("X") was presented, and the participants were required to press the correct key as soon as possible. The $\mathrm{X}$ was presented 
for the remaining duration of the trial with a minimum presentation time of $100 \mathrm{~ms}$. All participants continued to the next task when six test blocks had been completed.

\section{Fear Conditioning and Generalisation Protocol}

To quantify the extent to which global/local attention corresponded with differences in the generalisation of fear, participants were first trained to acquire fear towards one of two human faces, followed by the measurement of their fear generalisation to perceptually similar faces (Lissek et al. 2008; Lau et al. 2011). Prior to the commencement of this task, participants were told that they would see a series of faces and that some of these faces would be followed by a loud scream. Their job was to decide which faces were paired with the scream and to rate the extent to which they were afraid that the scream would occur on the scale presented to them in each trial.
Each trial of the experiment began with a fixation cross $($ ' +') for $2000 \mathrm{~ms}$ at the centre of the screen, followed by the CS or GS for $8000 \mathrm{~ms}$. In trials without the US, the CS/ GS was presented for a further $2000 \mathrm{~ms}$. In trials with a US, a loud scream $(103 \mathrm{db})$ was presented along with the same actor as the CS + face expressing a shocked reaction for $2000 \mathrm{~ms}$ (see Fig. 2). Upon stimulus offset, an intertrial-interval that was a random interval of between 1000 and $5000 \mathrm{~ms}$ was presented during which time the screen was blank and after which the next trial began. As such, there was an interval of between 5000 and 9000 ms between CS presentations. The scream used in this experiment was the same as that used in other 'screaming US' investigations (Lau et al. 2011). Lipp (2006) found that a scream at this volume is safe to use whilst producing a reliable fear response.

The experiment included two conditional stimuli: two Chinese male faces with neutral expressions selected from an existing database of faces developed at the University
Fig. 2 Fear acquisition measured on a self-reported fear rating and $\mathbf{b}$ skin conductance response (SCR) of participants in Global and Local attentional training groups in the fear acquisition phase. $\mathrm{NS}=\mathrm{Not}$ Significant; * significant at $\mathrm{p}<0.05 ; * *$ significant at $\mathrm{p}<0.005$, *** significant at $\mathrm{p}<0.001$. Standard error bars are presented
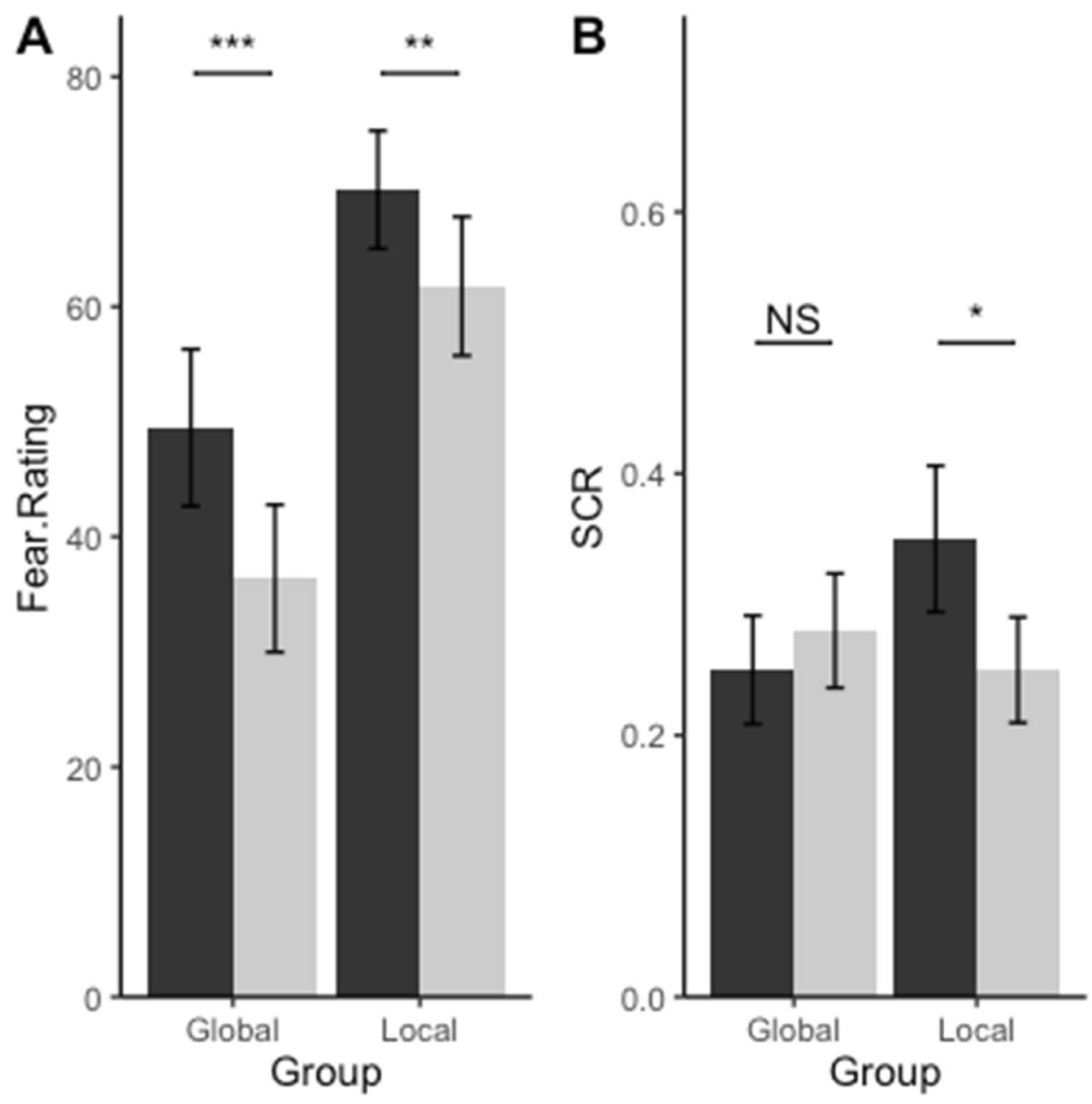

Stimulus.Type

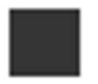

\section{CS-}


of Hong Kong (Zhang et al. 2017). One face was paired with the US (CS+) and one was not (CS-). The faces that were used for $\mathrm{CS}+$ and CS- were randomised between participants. Participants were also shown four additional faces or generalisation stimuli (GS) that were perceptual morphs positioned on a gradient between the CS + and CS-, at ratios of $80 \% \mathrm{CS}+/ 20 \% \mathrm{CS}$ - (GS1), $60 \% / 40 \%$ (GS2), $40 \% / 60 \%$ (GS3) and 20\%/80\% (GS4) (see Fig. 1).

The fear conditioning and generalisation protocol comprised of three phases: Habituation, Acquisition and Generalisation. During Habituation, the CS + and CS- faces were presented four times each without the US in order to reduce orienting responses to either of the stimuli. During the Acquisition phase, both stimuli were presented eight times each, and the CS + was followed by the US on six of these trials (75\% reinforcement). The CS- was never followed by the US. During the Generalisation phase, the CS +, CS- and GSs were presented four times each and, to prevent extinction, the CS + was reinforced on two of these trials (50\% reinforcement). Within each phase the trials were presented in a pseudo-random order such that trials involving the same stimuli were not presented more than two times successively.

\section{Self-Reported Fear}

During each trial in each phase (habituation, acquisition and generalisation) participants were instructed to click on the scale presented to them regarding how afraid they were of each face. Only the numbers 1 (not afraid at all) 100 (extremely afraid) were labelled on the scale. This scale appeared under each CS/GS $2000 \mathrm{~ms}$ after stimulus onset and disappeared after $6000 \mathrm{~ms}$, prior to when the US would occur, whether it occurred within that trial or not. The scale disappeared after this time, irrespective of whether participants made a response or not. If no response was made, the data were to be recorded as missing. There was no missing data recorded in the present investigation. Mean selfreported fear ratings for each stimulus in Acquisition and Generalisation were computed.

\section{Skin Conductance Responding (SCR)}

Throughout each trial participants' skin conductance response (SCR) was also recorded using BIOPAC MP150 hardware unit and AcqKnowledge version 4.2 software (BIOPAC Systems, Inc.). Participants' skin conductance level was detected by two EL507 $11 \mathrm{~mm}$ diameter $\mathrm{Ag} / \mathrm{AgCl}$ electrodes placed on the hypothenar eminence and inneredge of the palmar distal transverse of the palm of participants' non-dominant hand. The increase in conductivity between the two electrodes provided a skin conductance reading, sampled using one GSR100C amplifier and two LEAD110A at a rate of $31.25 \mathrm{~Hz}$ and filtered using a FIR low-pass filter at a fixed frequency cut-off of $2 \mathrm{~Hz}$. Participants' SCR in each trial was calculated by subtracting the mean skin conductance value at baseline during the $2000 \mathrm{~ms}$ prior to stimulus onset from the maximum skin conductance value during the $8000 \mathrm{~ms}$ that the CS/GS was on the screen but prior to the US. Negative responses were recoded as zero and participants' SCR values were subjected to withinparticipant range correction based on the maximum response of each participant to the US to account for inter-individual differences in responding and square root transformation to account for skew (Lonsdorf et al. 2017). Mean scores for the transformed SCRs were computed for each participant, for each stimulus within the Acquisition and Generalisation phases separately.

\section{Procedure}

All participants were invited to the laboratory where they first completed the DASS, followed by the attention training protocol, and then the fear conditioning and generalisation procedure.

\section{Data Processing and Analysis Procedure}

\section{Analysis Approach}

The analysis was divided into two parts. First, as trait levels of anxiety and other associated measures of psychopathology have been associated with differences in attentional breadth and fear generalisation (Derryberry \& Reed 2002; Lissek et al. 2014) group differences in baseline levels of depression, anxiety and stress were tested in order to establish that these variables did not differ between groups. Where there was evidence of a difference in these variables, these scores were included in subsequent analyses (no group differences emerged so these variables were not included in subsequent analyses). Second, we compared participants' reaction time (RT) to complete the trials between the first and the final block of attention training within the Global and Local groups as a manipulation check. Finally, we tested for differences between the Global and Local groups regarding the extent to which fear (self-reports and SCR) was acquired and generalised.

\section{Manipulation Check of Training Effects}

To test whether attention training successfully induced training-congruent attention breadth, we conducted two paired $t$-tests comparing the mean RT between the first and final (sixth) block of the training within the Global and Local groups. A lower mean RT in the final block of 
the attention training, compared to the first block, would indicate that the training successfully induced the trainingcongruent attention breadth by improving participants' speed at performing the Global or the Local trials.

\section{Between-Group Analyses of Training Effects}

To test for differences between groups in the extent to which fear was conditioned during Acquisition, average scores across the Acquisition phase trial blocks for SCR and self-reported fear levels were analysed in two 2 (stimulus type: $\mathrm{CS}+, \mathrm{CS}-) \times 2$ (group: Global, Local) mixed ANOVA. Fear conditioning would be represented either by a main effect of stimulus type, with $\mathrm{CS}+$ responding higher than CS- responding, or an interaction between stimulus type and group such that the difference between CS + and CS- differed between groups. Wilks' lambda was used to compute the ANOVA, and Greenhouse-Geisser corrections were performed.

Using a similar procedure, to test whether fear generalised and the extent to which this differed as a function of group, two separate 6 (stimulus type: CS-, GS1, GS2, GS3, GS4, CS +) $\times 2$ (group: Global, Local) mixed ANOVA for average SCR and fear ratings scores across the Generalisation phase trial blocks were performed. A main effect of group within these analyses would suggest that the groups differed in their overall fear across the six CS/GS, whereas a group by stimulus type interaction would indicate that the groups differed in the gradient of their generalisation with some stimuli being feared more than others between the groups. Where there was evidence of significant main effects or interaction effects, Bonferroni-corrected paired or independent samples t-tests were performed. Fear levels (self-report; SCR) were expected to be most different between the CS + and the most dissimilar GSs (e.g., GS3, GS4) and vice versa for comparisons of the GS relative to the CS-. The extent of these between-stimulus differences were also expected to differ between groups.

\section{Results}

\section{Baseline Check}

The Local and Global groups did not differ in self-reported levels of depression $(t(37)=0.06, p=0.955, d=0.018)$, anxiety $(t(37)=0.13, p=0.901, d=0.040)$, and stress $(t(37)=0.24, p=0.809, d=0.078$; see Table 1 and supplementary material).
Table 1 Descriptive of measured variables, mean and (SD) presented

\begin{tabular}{|c|c|c|}
\hline \multirow[t]{2}{*}{ Measure } & \multicolumn{2}{|l|}{ Mean (SD) } \\
\hline & $\begin{array}{l}\text { Global training group } \\
(\mathrm{N}=19 ; 6 \text { males, } 13 \\
\text { females })\end{array}$ & $\begin{array}{l}\text { Local training } \\
\text { group } \\
(\mathrm{N}=20 ; 8 \\
\text { males, } 12 \\
\text { females })\end{array}$ \\
\hline \multicolumn{3}{|l|}{ DASS } \\
\hline Depression subscale & $9.21(6.84)$ & $9.35(8.32)$ \\
\hline Anxiety subscale & $9.32(5.66)$ & $9.05(7.38)$ \\
\hline Stress subscale & $15.79(7.68)$ & $15.2(7.47)$ \\
\hline \multicolumn{3}{|l|}{ Fear acquisition } \\
\hline \multicolumn{3}{|l|}{ Fear rating } \\
\hline $\mathrm{CS}+$ & 49.48 (29.74) & $70.17(22.92)$ \\
\hline CS- & $36.38(27.86)$ & $61.78(27.01)$ \\
\hline \multicolumn{3}{|l|}{ SCR } \\
\hline $\mathrm{CS}+$ & $0.25(0.18)$ & $0.35(0.25)$ \\
\hline CS- & $0.28(0.19)$ & $0.25(0.18)$ \\
\hline \multicolumn{3}{|l|}{ Fear generalisation } \\
\hline \multicolumn{3}{|l|}{ Fear rating } \\
\hline $\mathrm{CS}+$ & $61.54(27.29)$ & $79.06(27.69)$ \\
\hline GS1 & $4704(29.70)$ & $73.47(27.11)$ \\
\hline GS2 & 42.68 (29.79) & $68.57(29.33)$ \\
\hline GS3 & $40.35(29.89)$ & $63.6(30.22)$ \\
\hline GS4 & 40.5 (31.79) & $61.45(29.8)$ \\
\hline CS- & $54.53(28.08)$ & $78.53(26.12)$ \\
\hline \multicolumn{3}{|l|}{ SCR } \\
\hline $\mathrm{CS}+$ & $0.26(0.19)$ & $0.32(0.25)$ \\
\hline GS1 & $0.25(0.19)$ & $0.17(0.15)$ \\
\hline GS2 & $0.24(0.19)$ & $0.23(0.19)$ \\
\hline GS3 & $0.17(0.16)$ & $0.19(0.19)$ \\
\hline GS4 & $0.22(0.23)$ & $0.20(0.17)$ \\
\hline CS- & $0.23(0.19)$ & $0.30(0.19)$ \\
\hline
\end{tabular}

\section{Manipulation Check of Training Effects}

Within the Global group, participants demonstrated increased speed (in milliseconds) at performing the final block $(M=550.02, S D=315.61)$ of the attention training than the first block $(M=635.55, S D=510.68)$, $t(911)=4.36, p<0.001, d=0.144$. Similarly, Local group participants also showed significantly higher speed at completing the final attention training block $(M=507.08, S D=207.71)$ compared to the first training block $(M=556.69, S D=398.08), t(959)=3.53, p<0.001$, $d=0.114$. Therefore, the training successfully induced training-congruent attentional breadth in participants in both training groups by improving their efficiency at completing the training trials. 
Between-Group Analyses of Training Effects

\section{Fear Acquisition}

\section{Self-Reported fear}

The ANOVA for differences in self-reported fear between $\mathrm{CS}+$ and $\mathrm{CS}-$, between the Local and Global training groups, showed main effects for stimulus type, $F(1$, $37)=15.29, p<0.001, \eta_{\mathrm{G}}{ }^{2}=0.051$, and group, $F(1$, $37)=10.61, p=0.002, \eta_{\mathrm{G}}{ }^{2}=0.199$. The group by stimulus type interaction was not significant, $F(1,37)=0.73$, $p=0.397, \eta_{\mathrm{G}}{ }^{2}=0.002$ (see Fig. 2a). The main effect of stimulus type was explained by higher self-reported fear in $\mathrm{CS}+$ trials than CS- trials in both groups $\left(M_{C S+}=60.10\right.$, $\left.S D_{C S+}=28.28 ; M_{C S-}=49.41, S D_{C S-}=30.10\right)$. The main effect of group was explained by greater self-reported fear in the Local group compared to the Global group (see Fig. 3a).

\section{Skin Conductance Response}

The two-way ANOVA for SCR for the CS + and CS- stimuli between the Local and Global training groups showed no main effect of stimulus type, $F(1,37)=1.33, p=0.256$,
Fig. 3 Self-reported fear rating during a Fear Acquisition and $\mathbf{b}$ Fear Generalisation of participants in Global and Local attentional training groups. NS $=$ Not Significant $; *$ significant at $\mathrm{p}<0.05 ; * *$ significant at $\mathrm{p}<0.005, * * *$ significant at $\mathrm{p}<0.001$. Standard error bars are presented
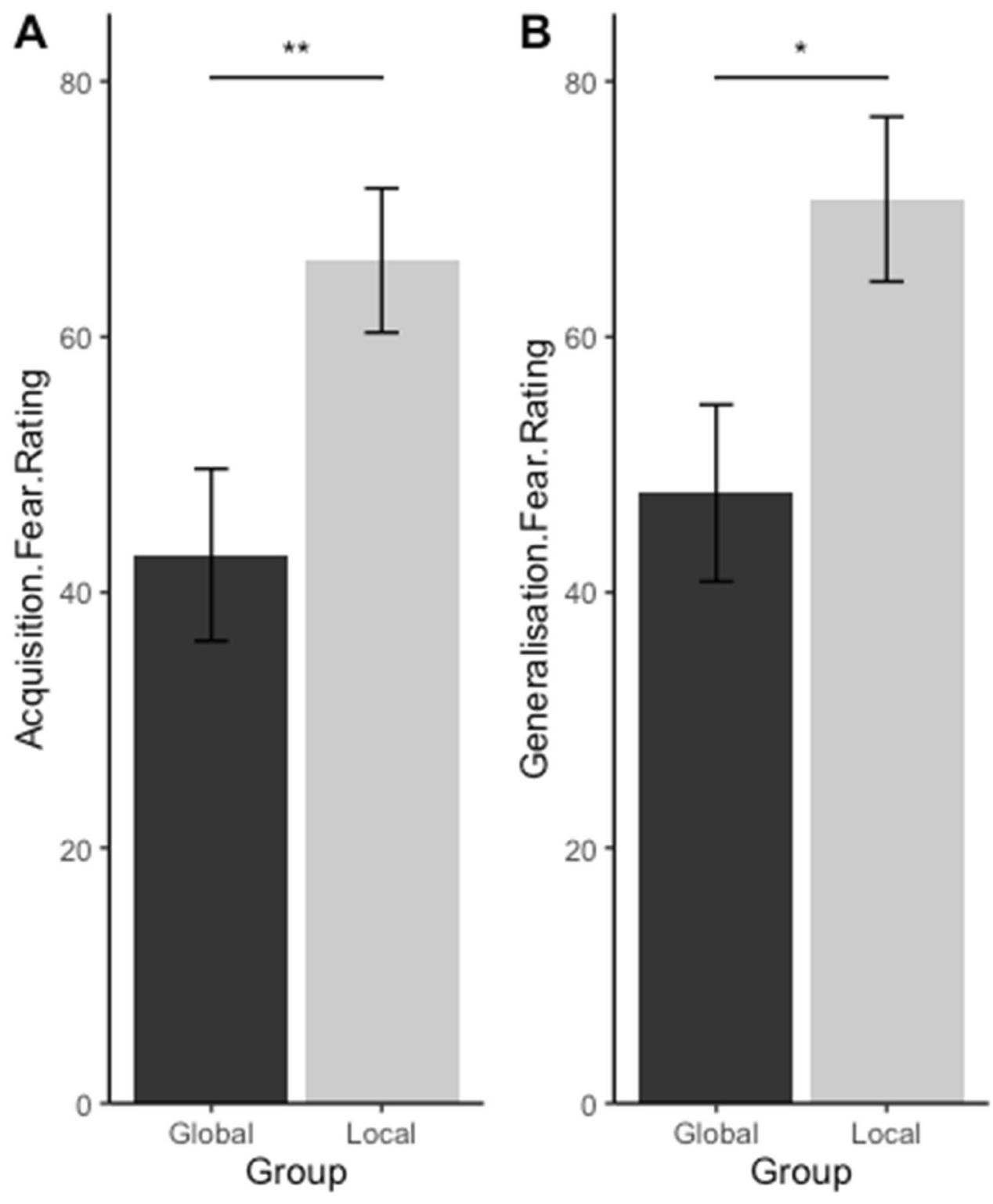

Group Global Local 
$\eta^{2}=0.016$, nor a main effect of group, $F(1,37)=0.654$, $p=0.424, \eta_{\mathrm{G}}{ }^{2}=0.009$ (see Table 1). However, a significant group by stimulus type interaction was observed, $F(1$, 37 ) $=4.128, p=0.049, \eta_{\mathrm{G}}{ }^{2}=0.049$ (see Fig. 2b). The group training by stimulus type interaction was explained by higher SCR in CS + trials than CS- trials in the Local group $\left(M_{C S+}=0.35, S D_{C S+}=0.25 ; M_{C S-}=0.25, S D_{C S-}=0.18\right.$; $t(39)=2.11, p=0.042, d=0.46)$ but not in the Global group $\left(M_{C S+}=0.25, S D_{C S+}=0.18 ; M_{C S-}=0.28, S D_{C S-}=0.19\right.$; $t(39)=0.79, p=0.429, d=0.15)$.

\section{Fear Generalisation}

\section{Self-Reported Fear}

The two-way ANOVA to test to what extent fear generalised on self-reported fear across the six levels of stimulus type (CS +, GS1, GS2, GS3, GS4, CS-), and the extent to which it differed between the Local and Global training groups revealed main effects for both stimulus type, $F(5,185)=25.95 p<0.001, \eta_{\mathrm{G}}{ }^{2}=0.062$ (see Fig. 4a), and group, $F(1,37)=6.96, p=0.012, \eta_{\mathrm{G}}{ }^{2}=0.145$ (see Fig. $3 b$ ). No group by stimulus type interaction was found, $F(5$, $185)=1.16, p=0.331, \eta_{\mathrm{G}}{ }^{2}=0.003$. The main effect of group was explained by significantly higher self-reported fear ratings in the Local group, compared to the Global group, for all the 6 levels of stimulus type (see Fig. 5a).

\section{A

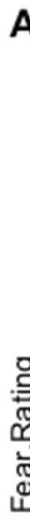
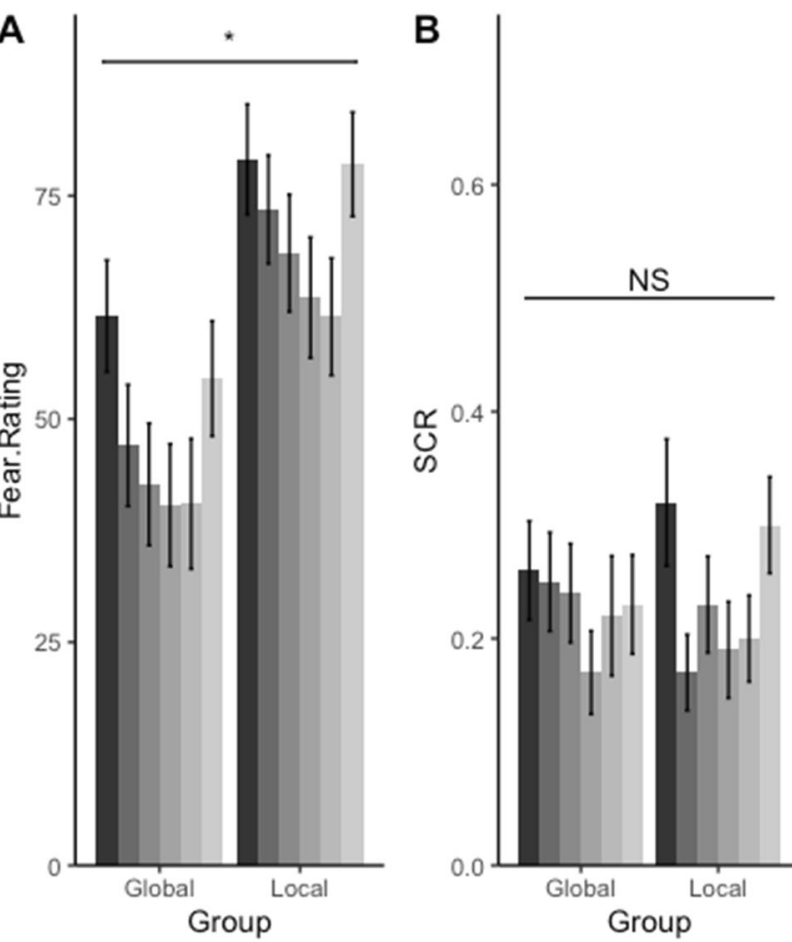

Stimulus.Type

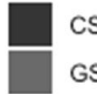

CS+
GS1

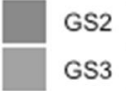

S3

GS4

CS-

Fig. 5 Fear generalisation measured on a self-reported fear rating and b skin conductance response (SCR) of participants in Global and Local attentional training groups in the fear generalisation phase. NS $=$ Not Significant; $*$ significant at $\mathrm{p}<0.05$; ** significant at $\mathrm{p}<0.005$, *** significant at $\mathrm{p}<0.001$. Standard error bars are presented
Fig. 4 Fear generalisation measured on a self-reported fear rating and $\mathbf{b}$ skin conductance response (SCR) across participants on the six stimulus type in the fear generalisation phase. NS = Not Significant; * significant at $\mathrm{p}<0.05 ; * *$ significant at $\mathrm{p}<0.005$, *** significant at $\mathrm{p}<0.001$. Standard error bars are presented
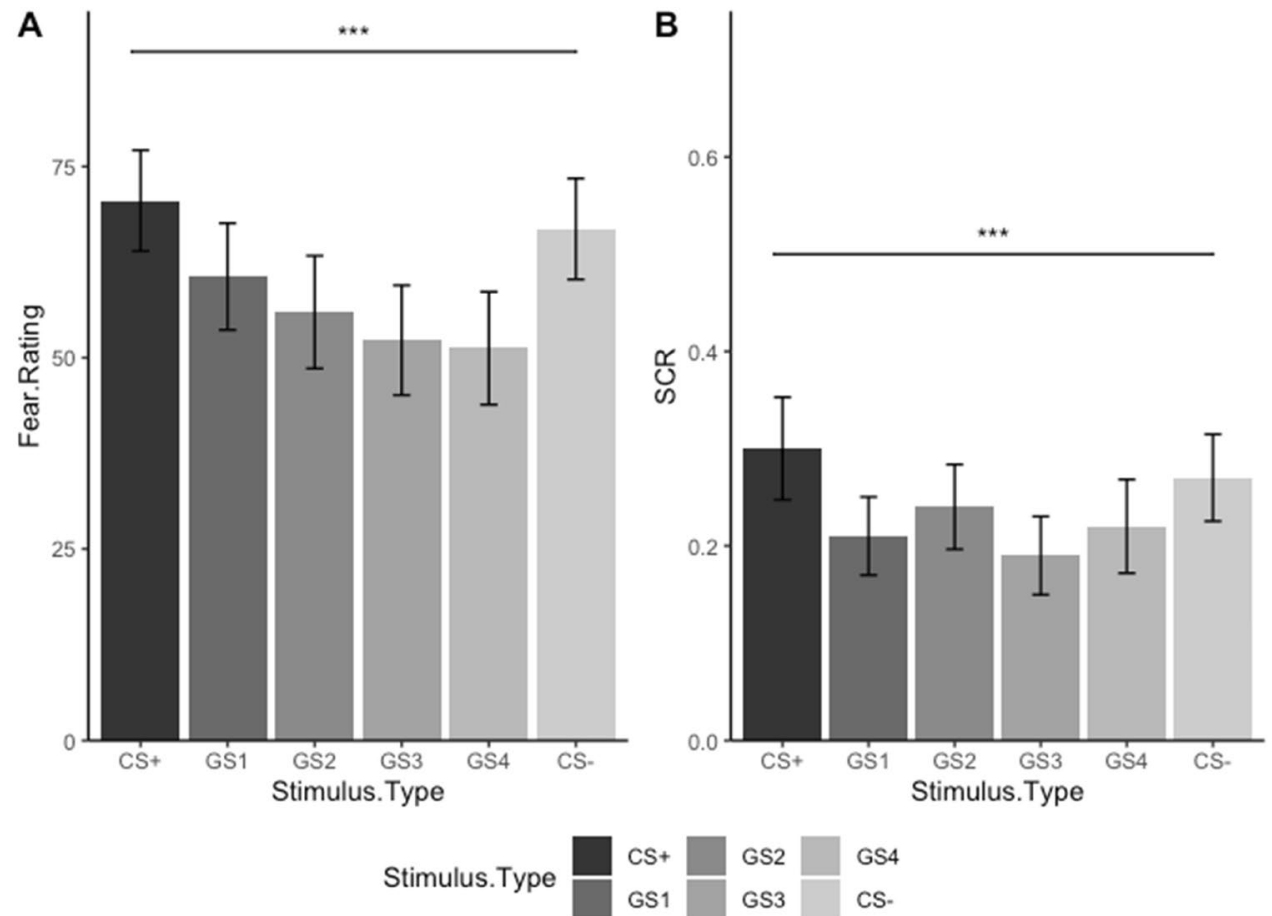


\section{Skin Conductance Response}

The ANOVA for generalisation of SCR for the six levels of stimulus type (CS +, GS1, GS2, GS3, GS4, CS-) and whether it differed between the Local and Global groups showed only a main effect of stimulus type, $F(5$, $185)=4.528, p<0.001, \eta_{\mathrm{G}}{ }^{2}=0.051$ (see Fig. 4b). No main effect of group was found, $F(1,37)=0.03, p=0.856$, $\eta_{\mathrm{G}}{ }^{2}<0.001$ (see Fig. 5b). A non-significant interaction effect between group and stimulus type was noted, $F(5$, $185)=2.18, p=0.058, \eta_{\mathrm{G}}{ }^{2}=0.025$.

\section{Discussion}

The present investigation examined the effects of attentional breadth on fear generalisation. Participants were trained to attend in either a global (holistic) way or a local (detail-oriented) way, after which they were conditioned to fear a stimulus and their generalisation of that fear was then measured. Participants in the Local attentional training group were expected to focus their attention so narrowly that they would pay particular attention to the unique features of the CSs and GSs and so more easily discriminate between them than participants in the Global attentional training group who were attending in a global manner, hence, were expected to attend only to the gist of each stimulus (e.g., that the faces were of dark-haired men). Therefore, compared to the Local attentional training group, participants in the Global attentional training group were expected to show greater generalisation of conditioned fear to perceptually similar generalisation stimuli due to a lack of focus on details.

Contrary to our hypothesis, during the generalisation phase participants in the Local training group demonstrated increased levels of self-reported fear to all stimuli relative to the Global training group. No such difference in the fear magnitude on skin conductance response was found between the two training groups. Our findings contribute to existing studies that have examined the correlation between individual differences in attentional focus (e.g., towards or away from threats; Baker et al. 2019) or which have modified attention using instructions (Vervliet et al. 2010). The findings presented here additionally suggest that the breadth of attentional focus might also influence the generalisation of fear, as evidenced by significantly higher self-reported fear ratings for GSs by the Local group.

Overall, the results indicated that participants in both attention training groups were able to acquire self-reported fear towards the aversively reinforced stimulus $(\mathrm{CS}+)$ and generalise their self-reported fear along a gradient of perceptual similarity where fear declined as the presented stimuli diverged from the conditional stimulus. However, during the acquisition phase, the Local group reported elevated fear ratings for both stimuli (CS +, CS-) compared to the Global group. This is the first experimental evidence that training people to focus their attention in a narrow or local way may elevate their levels of conditioned fear relative to training that broadens attention. Although this finding was not initially expected, it nonetheless relates to those of other studies that have demonstrated a positive association between focused attention to local perceptual features and negative emotions such as fear (Finucane \& Power 2010), anxiety (Derryberry \& Reed 1998, 2002), and incidental negative mood (Gasper \& Clore 2002). Our findings combined with the findings of other research studies lend support to a potential two-way causal route between narrowed focus of attention and fear and anxiety. Put otherwise, being put in fearful situations may prompt us to use a local attention style in order to successfully detect potential dangers in our environment, but a heightened tendency to focus attention on local features may also upregulate fear responses to potential dangers (e.g. faces presented as generalisation stimuli in the fear-conditioning paradigm).

During the acquisition phase, elevated fear levels for the $\mathrm{CS}+$ relative to the safe CS- were found to be significant for both groups only in participants' self-report ratings whereas for the electrodermal responses, only the Local group showed discrimination between the CS + and CSAlthough habituation of SCR responses is common and can occur rapidly (Boucsein et al. 2012), another explanation for the non-significant difference between CS + and CS- in the Global group may be that the global attentional training weakened participants' perceptual discrimination of the CS + and CS- face. Previous research has shown that a global attention style is associated with reduced ability to perceptually discriminate between face stimuli (e.g., Chen et al. 2002). In our study being trained to holistically process visual stimuli may have reduced the ability of participants to distinguish between the CS + and CS- faces, thereby contaminating SCR measurement of acquisition. Moreover, both face stimuli were of Chinese males and presented in the same context (i.e. against a black screen) which could also have contributed to the difficulty amongst Global group participants to perceptually discriminate between the two stimuli, again contaminating differences between $\mathrm{CS}+$ and CS- (Byrom \& Murphy 2018). Future studies might want to utilise a second, unrelated, CS- (e.g., a shape) or separate dangerous and safe contexts (Mühlberger et al. 2014) to test these hypotheses. Such investigations would allow researchers to discern between fear that is felt for safe stimuli or contexts that have no association with the original conditioning versus those that are perceptually related to them and so are susceptible to generalisation effects.

During the generalisation phase, both groups showed a linear decrement in self-reported fear ratings between the CS +, GSs and the CS-. However, the Local group 
demonstrated increased magnitude of fear generalisation, as indicated by higher self-reported fear ratings for all stimuli (CS + , GSs, CS-) compared to the Global group. This novel finding is in line with previous research on the positive link between narrowed attention and anxiety (e.g., Derryberry \& Reed 1998, 2002) and extends the effect of attention on fear generalisation by experimentally demonstrating that local or narrow attentional training may causally elevate the magnitude of fear generalization compared to global or holistic attentional training. The increased magnitude of selfreported fear ratings in the Local group could be because, during the acquisition phase, they acquired a higher degree of fear to both $\mathrm{CS}+$ and CS- following the attention training. Follow-up studies could dissociate between whether attention training directly influenced acquisition (indirectly increasing generalisation) or generalisation by comparing the results obtained from presenting the attentional training paradigm before fear acquisition and generalisation with presenting the training after acquisition and before generalisation. This distinction would help in the development of feasible interventions for anxiety disorders where a global attentional style is trained prior to or immediately following an aversive experience in order to reduce the subsequent generalisation of fear to other situations or stimuli in life.

We did not observe differences in fear generalisation magnitude between the two attention training groups on SCR during the generalisation phase. This difference in findings between SCR and self-reported fear ratings is perhaps not surprising as self-report and physiological measures of fear are thought to reflect different aspects of fear and so do not often follow the same pattern of responding (Beckers et al. 2013). Therefore, we speculate that our findings may indicate that although participants in the Global group are able to demonstrate enhanced cognitive discrimination between the CS + and the other stimuli, compared to Local group participants; but on a more automatic level both groups may respond as though the $\mathrm{CS}+$ is equivalent to the other, safe, stimuli that were presented. Future investigations should use additional measures of autonomic fear responses, such as fear potentiated startle, to test whether the two training groups differ on their ability to perceptually discriminate based on their autonomic fear indices for dangerous and safe stimuli. Future research using eye-tracking and neuroimaging techniques are also needed in order to map the neurocognitive mechanisms underlying the difference in fear generalisation between the Local and Global attention training groups. Conducting an eye-tracking study would also reveal the extent to which the training actually modified attentional breadth.

To conclude, the present study offers novel insights into the effects of attentional breadth on fear generalisation. Training people to attend to stimuli in a local or detailed manner leads to greater fear for both dangerous and safe stimuli compared to a person trained to attend to stimuli in a global or holistic manner. This finding of narrowed attentional focus could increase fear responses bears important clinical relevance in development of attention-based training interventions for the treatment of anxiety disorders and phobias. Given that the current research is in its infancy, therefore future research should focus on the neurocognitive mechanisms underlying the link between attentional breadth and fear learning/generalization. For instance, future work could compare the causal effect of experimentally-induced narrowed focus of attention vs. heightened fear or state anxiety to test whether a two-way causal route exists between local attention and fear/anxiety. Future experimental work could also test whether there are differences in the effects of the attention training depending on whether it is administered prior to or following fear acquisition. Furthermore, a recent study showed that the cognitive process of mental imagery can induce conditioned avoidance within a fear conditioning paradigm when the association between a neutral stimulus and an aversive response (e.g. electric shock) are imagined (Krypotos et al. 2020). Given that attentional breadth can modulate the focus of attention, future research could also explore the extent to which attentional breadth modulates attention during mental imagery in order to influence major symptoms of anxiety-related disorders, such as heightened fear generalization and behavioural avoidance of fear-related stimuli.

Funding This research was supported by a seed grant from the first author's institution (Project code: 201703159003).

\section{Compliance with Ethical Standards}

Research Involving Human and Animal Participants All procedures performed in the study involved human participants and were in accordance with the ethical standards of the institutional and/or national research committee and with the 1964 Helsinki declaration and its later amendments or comparable ethical standards. No animal studies were conducted in this study.

Informed Consent Informed consent was obtained from all individual participants included in the study.

Open Access This article is licensed under a Creative Commons Attribution 4.0 International License, which permits use, sharing, adaptation, distribution and reproduction in any medium or format, as long as you give appropriate credit to the original author(s) and the source, provide a link to the Creative Commons licence, and indicate if changes were made. The images or other third party material in this article are included in the article's Creative Commons licence, unless indicated otherwise in a credit line to the material. If material is not included in the article's Creative Commons licence and your intended use is not permitted by statutory regulation or exceeds the permitted use, you will need to obtain permission directly from the copyright holder. To view a copy of this licence, visit http://creativecommons.org/licenses/by/4.0/. 


\section{References}

Ahrens, L. M., Mühlberger, A., Pauli, P., \& Wieser, M. J. (2015). Impaired visuocortical discrimination learning of socially conditioned stimuli in social anxiety. Social cognitive and affective neuroscience, 10(7), 929-937.

Ahrens, L. M., Pauli, P., Reif, A., Mühlberger, A., Langs, G., Aalderink, T., et al. (2016). Fear conditioning and stimulus generalization in patients with social anxiety disorder. Journal of Anxiety Disorders, 44, 36-46.

Baker, H. M., Barry, T. J., Kumari, V., Pandey, R., Shanta, N., \& Lau, J. Y. F. (2019). Problematic attention processing and fear learning in adolescent anxiety: Testing a combined cognitive and learning processes model. Journal of Behavior Therapy and Experimental Psychiatry, 62, 146-153.

Barry, T. J., Griffith, J. W., Vervliet, B., \& Hermans, D. (2016a). The role of stimulus specificity and attention in the generalization of extinction. Journal of Experimental Psychopathology, 7(1), 143-152. https://doi.org/10.5127/jep.048615.

Barry, T. J., Vervliet, B., \& Hermans, D. (2016b). Threat-related gaze fixation and its relationship with the speed and generalisability of extinction learning. Australian Journal of Psychology, 68(3), 200-208. https://doi.org/10.1111/ajpy.12124.

Barry, T. J., Vervliet, B., \& Hermans, D. (2017). Feature specific attention and return of fear after extinction. Journal of Experimental Psychopathology, 8(1), 76-87. https://doi.org/10.5127/jep.05111 5.

Beckers, T., Krypotos, A. M., Boddez, Y., Effting, M., \& Kindt, M. (2013). What's wrong with fear conditioning? Biological Psychology, 92(1), 90-96.

Boucsein, W., et al. (2012). Publication recommendations for electrodermal measurements. Psychophysiology, 49, 1017-1034.

Byrom, N. C., \& Murphy, R. A. (2018). Individual differences are more than a gene $\mathrm{x}$ environment interaction: The role of learning. $J$ Exp Psychol Anim Learn Cogn, 44(1), 36-55. https://doi.org/10.1037/ xan0000157.

Chen, Y. P., Ehlers, A., Clark, D. M., \& Mansell, W. (2002). Patients with generalized social phobia direct their attention away from faces. Behaviour Research and Therapy, 40(6), 677-687. https:// doi.org/10.1016/S0005-7967(01)00086-9.

Chuk, T., Chan, A. B., \& Hsiao, J. H. (2014). Understanding eye movements in face recognition using hidden Markov models. Journal of Vision, 14(11), 8-8. https://doi.org/10.1167/14.11.8.

Derryberry, D., \& Reed, M. A. (1998). Anxiety and attentional focusing: trait, state and hemispheric influences. Personality and Individual Differences, 25(4), 745-761.

Derryberry, D., \& Reed, M. A. (2002). Anxiety-related attentional biases and their regulation by attentional control. Journal of Abnormal Psychology, 111(2), 225-236. https://doi. org/10.1037/0021-843X.111.2.225.

Dunsmoor, J. E., Mitroff, S. R., \& LaBar, K. S. (2009). Generalization of conditioned fear along a dimension of increasing fear intensity. Learning \& Memory, 16(7), 460-469.

Dunsmoor, J. E., \& Paz, R. (2015). Fear generalization and anxiety: behavioral and neural mechanisms. Biological psychiatry, 78(5), 336-343.

Dymond, S., Dunsmoor, J. E., Vervliet, B., Roche, B., \& Hermans, D. (2015). Fear generalization in humans: systematic review and implications for anxiety disorder research. Behavior Therapy, 46(5), 561-582.

Finucane, A. M., \& Power, M. J. (2010). The effect of fear on attentional processing in a sample of healthy females. Journal of Anxiety Disorders, 24(1), 42-48.
Gasper, K., \& Clore, G. L. (2002). Attending to the big picture: Mood and global versus local processing of visual information. Psychological Science, 13(1), 34-40.

Ginat-Frolich, R., Gendler, T., Marzan, D., Tsuk, Y., \& Shechner, T. (2019). Reducing fear overgeneralization in children using a novel perceptual discrimination task. Behaviour Research and Therapy, 116, 131-139. https://doi.org/10.1016/j.brat.2019.03.008.

Ginat-Frolich, R., Klein, Z., Katz, O., \& Shechner, T. (2017). A novel perceptual discrimination training task: Reducing fear overgeneralization in the context of fear learning. Behaviour Research and Therapy, 93, 29-37. https://doi.org/10.1016/j.brat.2017.03.010.

Holt, D. J., Boeke, E. A., Wolthusen, R. P. F., Nasr, S., Milad, M. R., \& Tootell, R. B. H. (2014). A parametric study of fear generalization to faces and non-face objects: Relationship to discrimination thresholds. Frontiers in Human Neuroscience. https://doi. org/10.3389/fnhum.2014.00624.

Hovland, C. I. (1937). The generalization of conditioned responses: I The sensory generalization of conditioned responses with varying frequencies of tone. The Journal of General Psychology, 17(1), $125-148$.

Kaczkurkin, A. N., Burton, P. C., Chazin, S. M., Manbeck, A. B., Espensen-Sturges, T., Cooper, S. E., et al. (2017). Neural substrates of overgeneralized conditioned fear in PTSD. American Journal of Psychiatry, 174(2), 125-134. https://doi.org/10.1176/ appi.ajp.2016.15121549.

Krypotos, A. M., Mertens, G., Leer, A., \& Engelhard, I. M. (2020). Induction of conditioned avoidance via mental imagery. Behaviour Research and Therapy, 132, 103652.

Lau, J. Y., Britton, J. C., Nelson, E. E., Angold, A., Ernst, M., Goldwin, M., et al. (2011). Distinct neural signatures of threat learning in adolescents and adults. Proceedings of the National Academy of Sciences, 108, 4500-4505.

Laufer, O., Israeli, D., \& Paz, R. (2016). Behavioral and neural mechanisms of overgeneralization in anxiety. Current Biology, 26(6), 713-722. https://doi.org/10.1016/j.cub.2016.01.023.

Lipp, O. V. (2006). Of snakes and flowers: Does preferential detection of pictures of fear-relevant animals in visual search reflect on fearrelevance? Emotion, 6(2), 296.

Lissek, S., Biggs, A. L., Rabin, S. J., Cornwell, B. R., Alvarez, R. P., Pine, D. S., et al. (2008). Generalization of conditioned fearpotentiated startle in humans: Experimental validation and clinical relevance. Behaviour Research and Therapy, 46(5), 678-687.

Lissek, S., Kaczkurkin, A. N., Rabin, S., Geraci, M., Pine, D. S., \& Grillon, C. (2014). Generalized anxiety disorder is associated with overgeneralization of classically conditioned fear. Biological Psychiatry, 75(11), 909-915. https://doi.org/10.1016/j.biops ych.2013.07.025.

Lonsdorf, T. B., Menz, M. M., Andreatta, M., Fullana, M. A., Golkar, A., Haaker, J., et al. (2017). Don't fear 'fear conditioning': methodological considerations for the design and analysis of studies on human fear acquisition, extinction, and return of fear. $\mathrm{Neu}$ roscience and Biobehavioral Reviews, 77, 247-285. https://doi. org/10.1016/j.neubiorev.2017.02.026.

Lovibond, P. F., \& Lovibond, S. H. (1995). The structure of negative emotional states: Comparison of the depression anxiety stress scales (DASS) with the beck depression and anxiety inventories. Behaviour Research and Therapy, 33(3), 335-343.

Mühlberger, A., Andreatta, M., Ewald, H., Glotzbach-Schoon, E., Tröger, C., Baumann, C., et al. (2014). The BDNF Val66Met Polymorphism modulates the generalization of cued fear responses to a novel context. Neuropsychopharmacology, 39(5), 1187-1195. https://doi.org/10.1038/npp.2013.320.

Navon, D. (1977). Forest before trees: The precedence of global features in visual perception. Cognitive Psychology, 9(3), 353-383. https://doi.org/10.1016/0010-0285(77)90012-3. 
Pomerantz, J. R. (1983). Global and local precedence: selective attention in form and motion perception. Journal of Experimental Psychology: General, 112(4), 516-540. https://doi. org/10.1037/0096-3445.112.4.516.

Schiele, M. A., Reinhard, J., Reif, A., Domschke, K., Romanos, M., Deckert, J., et al. (2016). Developmental aspects of fear: Comparing the acquisition and generalization of conditioned fear in children and adults. Developmental Psychobiology, 58(4), 471-481. https://doi.org/10.1002/dev.21393.

Staples-Bradley, L. K., Treanor, M., \& Craske, M. G. (2018). Discrimination between safe and unsafe stimuli mediates the relationship between trait anxiety and return of fear. Cognition and Emotion, 32(1), 167-173. https://doi.org/10.1080/02699931.2016.1265485.

Struyf, D., Zaman, J., Hermans, D., \& Vervliet, B. (2017). Gradients of fear: How perception influences fear generalization. Behaviour Research and Therapy, 93, 116-122. https://doi.org/10.1016/j. brat.2017.04.001.

Struyf, D., Zaman, J., Vervliet, B., \& Van Diest, I. (2015). Perceptual discrimination in fear generalization: Mechanistic and clinical implications. Neuroscience \& Biobehavioral Reviews, 59, 201207. https://doi.org/10.1016/j.neubiorev.2015.11.004.
Vervliet, B., Kindt, M., Vansteenwegen, D., \& Hermans, D. (2010). Fear generalization in humans: Impact of verbal instructions. Behaviour Research and Therapy, 48(1), 38-43.

Yoon, K. L., Vidaurri, D. N., Joormann, J., \& De Raedt, R. (2015). Social anxiety and narrowed attentional breadth toward faces. Emotion, 15(6), 682-686. https://doi.org/10.1037/emo0000070.

Zaman, J., Ceulemans, E., Hermans, D., \& Beckers, T. (2019). Direct and indirect effects of perception on generalization gradients. Behaviour Research and Therapy. https://doi.org/10.1016/j. brat.2019.01.006.

Zhang, J., Chan, A. B., Lau, E. Y., \& Hsiao, J. H. (2017). Insomniacs Misidentify Angry Faces as Fearful Faces Because of Missing the Eyes: An Eye-Tracking Study. Proceeding of the 39th Annual Conference of the Cogntive Science Society (pp. 1430-1435). Austin, TX: Cognitive Science Society.

Publisher's Note Springer Nature remains neutral with regard to jurisdictional claims in published maps and institutional affiliations. 